\title{
Interactive Thickness Visualization of Articular Cartilage
}

\author{
Matej Mlejnek* \\ ICGA \\ Vienna University of Technology
}

\author{
Anna Vilanova \\ Department of Biomedical Engineering \\ Eindhoven University of Technology
}

\author{
Meister Eduard Gröller* \\ ICGA \\ Vienna University of Technology
}

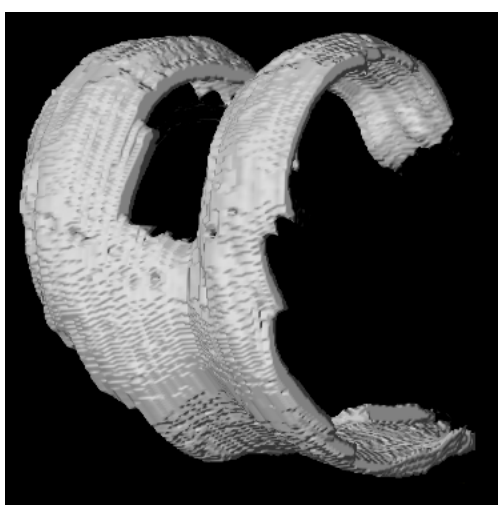

(a)

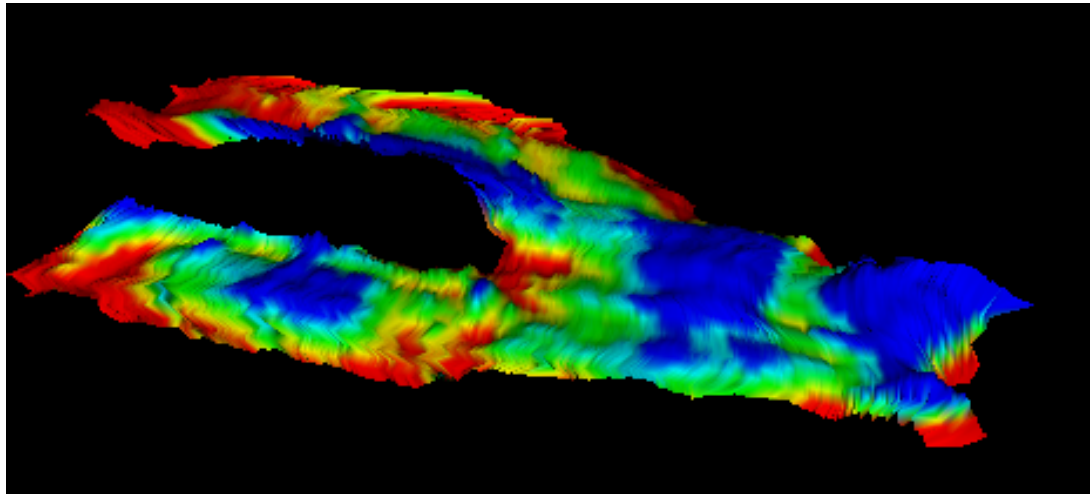

(b)

Figure 1: Surface reconstruction of articular cartilage from an MRI scan (a), and thickness height-field of the unfolded tissue (b).

\section{Abstract}

This paper describes a method to visualize the thickness of curved thin objects. Given the MRI volume data of articular cartilage, medical doctors investigate pathological changes of the thickness. Since the tissue is very thin, it is impossible to reliably map the thickness information by direct volume rendering. Our idea is based on unfolding of such structures preserving their thickness. This allows to perform anisotropic geometrical operations (e.g., scaling the thickness). However, flattening of a curved structure implies a distortion of its surface. The distortion problem is alleviated through a focusand-context minimization approach. Distortion is smallest close to a focal point which can be interactively selected by the user.

CR Categories: I.3.8 [Computing Methodologies]: Computer Graphics-Applications; J.3 [Computer Applications]: Life and medical sciences-Medical information systems

Keywords: visualization in medicine, applications of visualization

\section{Introduction AND MEdical BACKGROUND}

The surfaces of knee joints are covered by tissue with a complex structure, called articular cartilage. The main functions of the cartilage include distribution of weight, frictionless motion, and shock absorption. The most common cause of articular cartilage lesion is osteoarthritis. The degenerative process starts with tissue softening, and continues through swelling and thinning up to ulceration with exposure of the underlying bone. The development of osteoarthritis may take several years, however, at some point it is an irreversible

*e-mail:mlejnek@cg.tuwien.ac.at

†e-mail:a.vilanova@tue.nl

‡email:groeller@cg.tuwien.ac.at process. If the diagnosis of the osteoarthritis is determined too late, a painful stiffness and a limitation of the joint's function may result $[6,13,10]$. Since the cartilage is only a few millimeters thick, an accurate measurement of the thickness is necessary for the early determination of a joint's degeneration. Two measures, i.e., volume and thickness of the cartilage, are typically used for a quantitative characterization of the cartilage. A change in cartilage thickness indicates the progress of the disease and can be used, e.g., for the estimation of the progress of osteoarthritis, or for the evaluation of the response to therapies.

Nowadays MR scanners and pulse sequences are very well capable of imaging cartilage and allow the assessment of its quality. Spatial perception is considerably reduced when viewing the MR volume $(512 \times 512 \times 50$ resolution used in clinical practice) slice by slice or by multi-planar reconstruction. This makes reading of the data by the radiologist unnecessarily difficult and prolongs the examination time. Moreover, the articular cartilage is a curved structure. Thereby, reading of the thickness changes from a direct volume rendered or a reconstructed surface model is quite difficult (see figure 1 (a)). Until now, the default technique for visualizing cartilage thickness has been color mapping. Williams et al. [23] visualized the cartilage thickness on the surface of the underlying bone.

Our approach to cartilage visualization deals with unfolding of the cartilage and depicting it as a height field (see figure 1 (b)). In comparison to direct volume rendering or surface reconstruction methods, the height field representation of the cartilage eliminates the complexity of the 3D shape of the cartilage. This allows the user to concentrate solely on the inspection of the cartilage thickness. The height field representation of the cartilage offers several visualization modes for representing the thickness information: color mapping, scaling, glyphs, iso-lines, etc. The entire cartilage is depicted at once, thus, giving an overview of the global thickness.

General surfaces cannot be flattened without some amount of distortion. The distortion can be reduced, or in some cases (e.g., developable surfaces) even eliminated by introducing cuts and seams. Such operations split the surface and introduce discontinuities, thus, losing spatial relations [19]. 


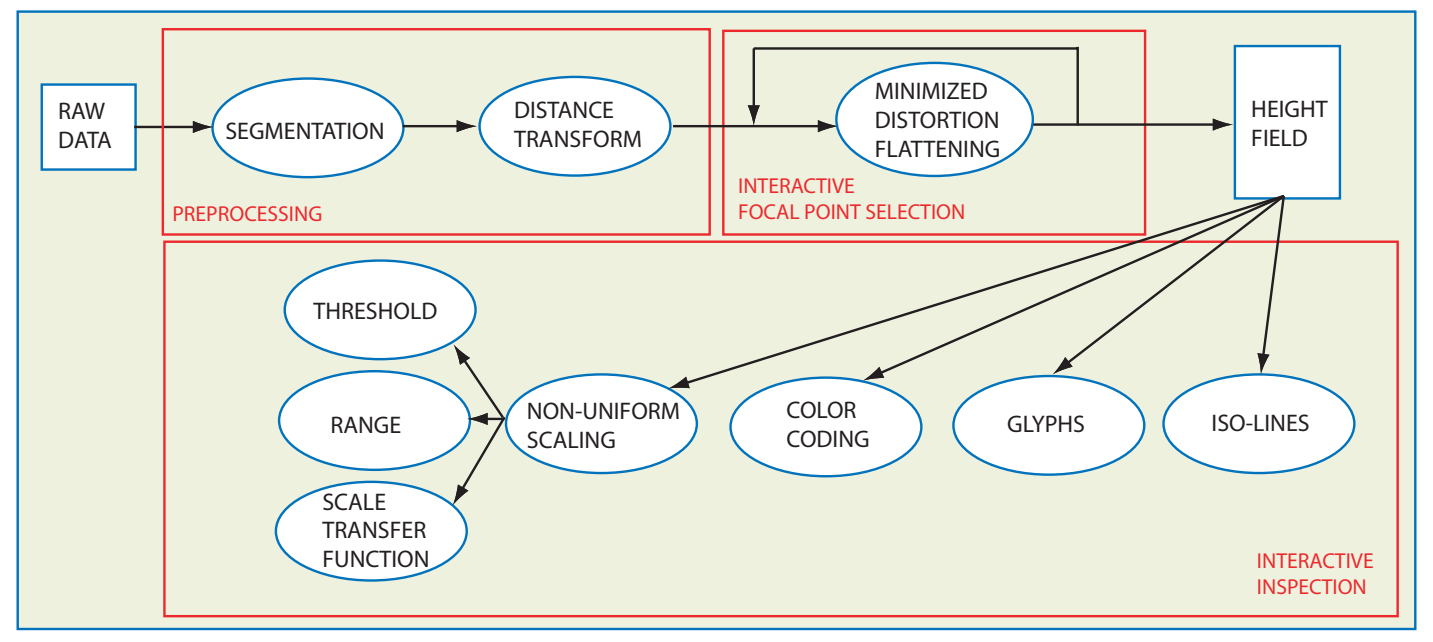

Figure 2: Pipeline for thickness visualization.

In our approach we locally minimize the distortion, in a userdefined area of interest (focus). The remaining part of the cartilage (context) is depicted in order to give an overview of the thickness of the entire cartilage. Since the overall thickness of the cartilage is different for each patient (according to the patient's body mass, age, sex, etc.), it is necessary to see the entire surface at once while inspecting it. The focal point on the surface of the cartilage can be interactively selected by the user.

The main contribution of this work is the handling and processing of articular cartilage as a typical thin-wall object. Another example would be colon walls. These objects have two dimensions with significant extent, whereas the third dimension (the thickness) is considerably smaller. The substantial difference in size requires the application of anisotropic operations (e.g., non-uniform scaling in thickness direction). We investigate surface flattening and discuss visualization techniques, which can be applied to the flattened surface.

Flattening requires the parameterization of the surface. In this respect a large body of work has been done for texture mapping purposes. The unfolding of anatomical structures has been discussed as an investigation tool in several areas of medical imaging, e.g., colon unfolding $[2,11]$, curved planar reformation [14], or flattening of the brain surface $[1,8]$. For these applications, the primary goal of the parameterization is usually the minimization of the global distortion over the entire surface. This process is time consuming and does not allow an interactive input from the user. In many medical applications, recent research has concentrated on detecting and investigating relatively small features. Therefore, we make use of local minimization of the distortion, preserving the shape and size of the area of interest, which can be interactively changed.

The paper is structured as follows. First, the visualization pipeline for thickness visualization will be sketched, describing the sequential stages in detail. Afterwards, an overview of operations on the resulting height field will be given in section 3. Finally, we summarize and conclude the work in section 4.

\section{Pipeline for Thickness Visualization}

The proposed pipeline for thickness visualization consists of the following steps (see figure 2). First, the raw volumetric data are semi-automatically segmented to identify the cartilage regions. In the second step, the distance between the inner and outer cartilage boundary is computed using a distance transform. Finally, the outer surface of the pre-segmented cartilage is triangulated using the min- imum edge criterion [7]. All these operations are done in the preprocessing step. The following steps are guided by the user who is provided with an immediate visual feedback. The flattening of the triangular mesh proposed in this paper is based on similar principles as the work by Sorkine et al. [22], however, we use different criteria for grading of the free vertices. We enable the user to interactively select a focal point, where the thickness shall be examined locally. The flattened mesh with assigned per-vertex thickness values corresponds to a height field with a triangulated base that consists of about $20 \mathrm{~K}$ triangles. Thus, the operations on the height field can be performed in real-time on commodity hardware. In the following, we will describe the pipeline steps in detail. The segmentation procedure is shortly described in section 2.1. Section 2.2 discusses the measurement of cartilage thickness, while in section 2.3 we explain the flattening of the surface.

\subsection{Cartilage Segmentation}

In order to describe the entire visualization pipeline, the segmentation process is shortly sketched in this section. However, the discussion on the segmentation of the cartilage is not significant to the contribution of the paper. In the literature, several approaches to cartilage segmentation have been discussed. Two main classes of segmentation methods are usually applied: manual segmentation and semi-automatic segmentation. If slice-by-slice segmentation is applied, it is usually carried out on sagittal slices. Along this direction, the topology of extracted cartilage contours does not radically change between the adjacent slices. Since the scanned MRI data are generally anisotropic, i.e., the in-plane voxel distance is smaller than the slice thickness, additional linearly interpolated contour slices are usually inserted between the adjacent slices.

Manual segmentation is usually time-consuming and requires an experienced user in order to obtain satisfactory results. Semiautomatic methods use thresholding, region growing, snakes, or edge detection filters in order to help the user in the segmentation.

To segment the cartilage from the MRI volume, we use an active contour model (snake) controlled by forces proposed by Lobregt et al. [17]. A snake, initially introduced by Kass et al. [15], is a parametric deformable contour. It is controlled by internal and external forces, which are usually defined in energy terms. The internal forces keep the snake smooth, while the external forces attract it to features, such as object boundaries.

The outcome of the segmentation is the contour of the cartilage in a slice. Due to our interest in the thickness of the tissue on the 


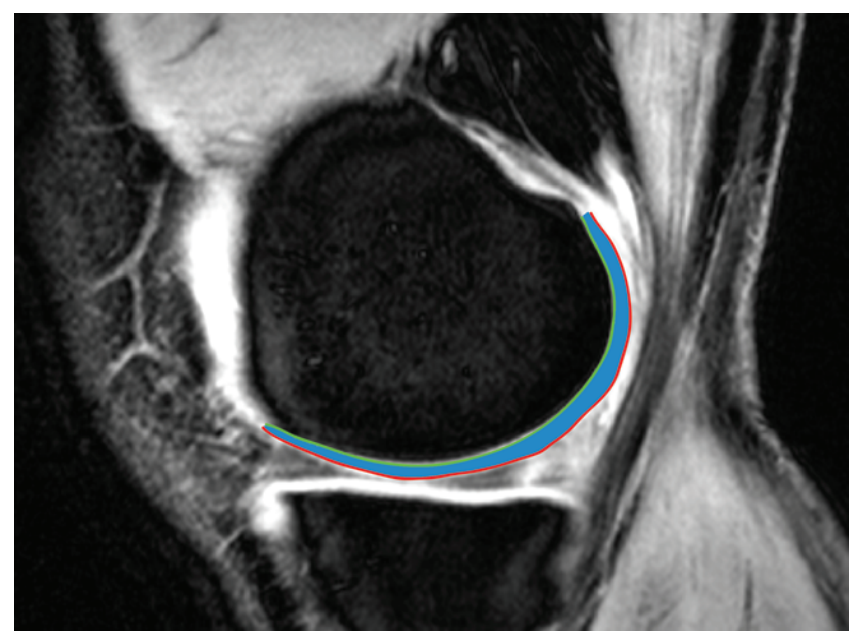

Figure 3: Sagittal slice of an MR scan of the knee joint. The cartilage is marked in blue with the inner boundary represented by the green line and the outer boundary shown by the red line.

outer surface, the contour is split into two parts (see figure 3). The first part is the inner boundary (green line) which is adjacent to the underlying bone. The second part of the contour is the outer boundary (red line) of the cartilage (blue). In the next step, we calculate the thickness from the outer to the inner boundary.

\subsection{Thickness Measurement}

There are several possibilities to calculate the thickness of a thin tissue. Heuer et al. [12] summarize three possibilities to measure cartilage thickness: vertical distance, proximity method (closest neighbor on the opposite surface), and normal distance (distance along the normal vector). For curved surfaces, the vertical distance metric is not appropriate since the computation of the distance is performed always along a constant (vertical) direction.

Applying the proximity method, we are looking for the Euclidean distance $D_{E}()$ between a point $p$ belonging to the outer boundary of the cartilage and the nearest point $r$ belonging to the inner boundary $I$.

$$
D_{E}(p)=\min \left(\sqrt{\left(p_{x}-r_{x}\right)^{2}+\left(p_{y}-r_{y}\right)^{2}+\left(p_{z}-r_{z}\right)^{2}}\right) ; \quad r \in I
$$

The distance computation can be efficiently approximated by the calculation of a distance field starting from the underlying bone. Recently, many optimizations of distance transforms have been discussed in the literature. They can be grouped into two categories: chamfer distance transforms and vector distance transforms [21]. The chamfer distance transform propagates the local distance by adding the neighborhood values, thus, propagating also the errors. On the other hand, the vector distance transform, introduced by Danielsson [5], propagates the distance vector to the nearest sample point of the object surface, thus, minimizing the average error. Vector distance transforms are in general slower than chamfer distance transforms. We are computing the distance field only for a thin cartilage layer close to the bone surface. Since we need an accurate measurement of the thickness, we use the computationally more expensive, but more accurate vector distance transform by Mullikin [18].

\subsection{Flattening of Articular Cartilage}

In order to perform unfolding of the cartilage, it is necessary to parameterize the outer boundary of the cartilage. Parameterizations of surfaces are often used in the area of texture mapping. In order to

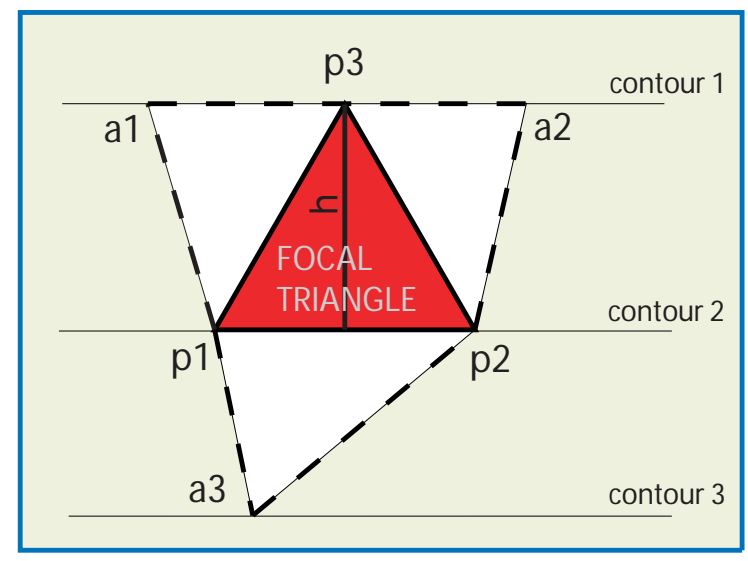

Figure 4: Surface flattening: The focal triangle $(p 1, p 2, p 3)$ is rigidly transformed to the patch. In the next step one point from the active set $\{a 1, a 2, a 3\}$ is chosen and added with the corresponding triangle to the mesh. This process is continued iteratively until all triangles are added to the patch.

measure the precision and faithfulness of the parameterization, several different metrics, e.g., based on preservation of lengths, angles, or areas, can be applied [3, 16, 20,9]. There are several parameters which can be adjusted in order to determine the trade-off between the different types of distortions and interactive frame rates. The setting of the parameters depends on the specific application.

For the purpose of flattening the curved surface of the cartilage into the corresponding 2D plane, the parameterization should fulfill the following criteria:

- We are interested not only in the thickness of the inspected part of the cartilage, but also in its size. Therefore, we need a parameterization, which minimizes area distortion. The ideal solution is an equiareal mapping.

- Local as well as global intersections have to be prevented this is a common problem in the area on surface parameterization.

- We do not allow multiple patches - the entire cartilage is rendered as one height field in order to keep spatial relations.

- Since the distortion cannot be eliminated for the entire patch, we allow interactive selection of an area of interest, where the distortion is primarily minimized.

- The parameterization has to be fast in order to allow interactive feedback.

As mentioned above, our parameterization technique has been inspired by the method presented by Sorkine et al. [22]. Since the cartilage contours are organized in planar slices, we need to prevent intersections of the contours also in parametric space. We deal with this problem in the following way. In order to efficiently prevent local as well as global intersections, we align all points belonging to one contour onto a line. This reduces the distortion minimization issue to a one dimensional optimization problem, thus, enabling reasonable frame rates.

In order to meet all of the above mentioned constraints we grow a planar patch around the selected triangle in the following manner. First, the focal triangle, the one which includes the focal point, is rigidly transformed into the $2 \mathrm{D}$ plane. Since the triangle vertices are arranged in planar contours, each triangle consists of two points $(p 1, p 2)$ belonging to one contour and the third one $(p 3)$ belonging 


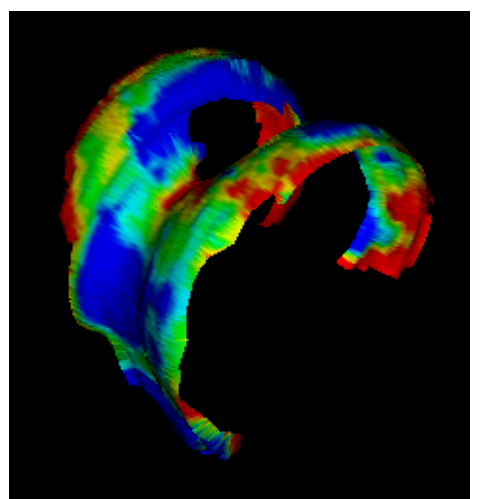

(a)

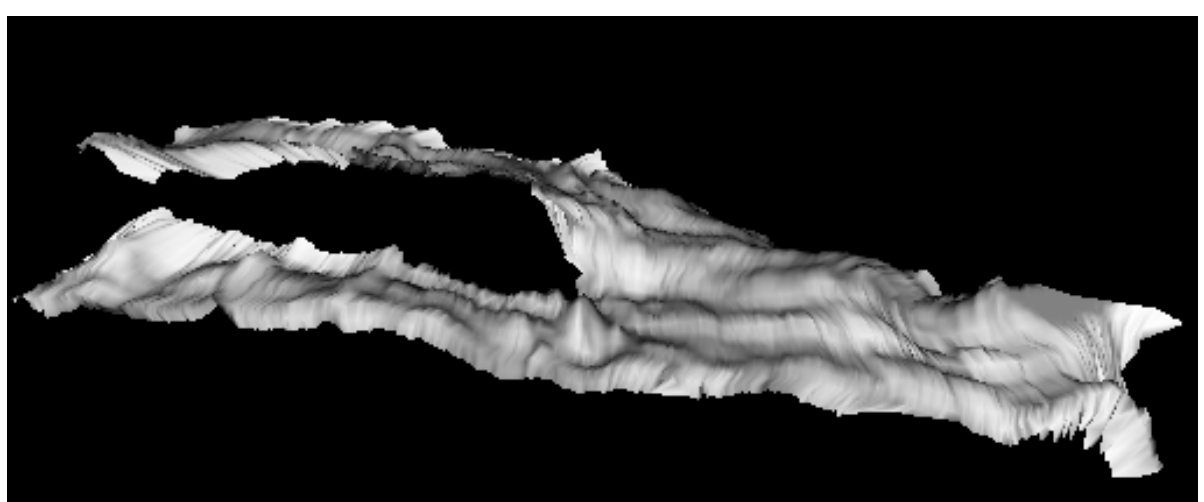

(b)

Figure 5: Color coded thickness on the reconstructed surface (a) and the height field representation with scale factor 3.0 (b). The "plastic" view of the cartilage offers an intuitive information about its thickness.

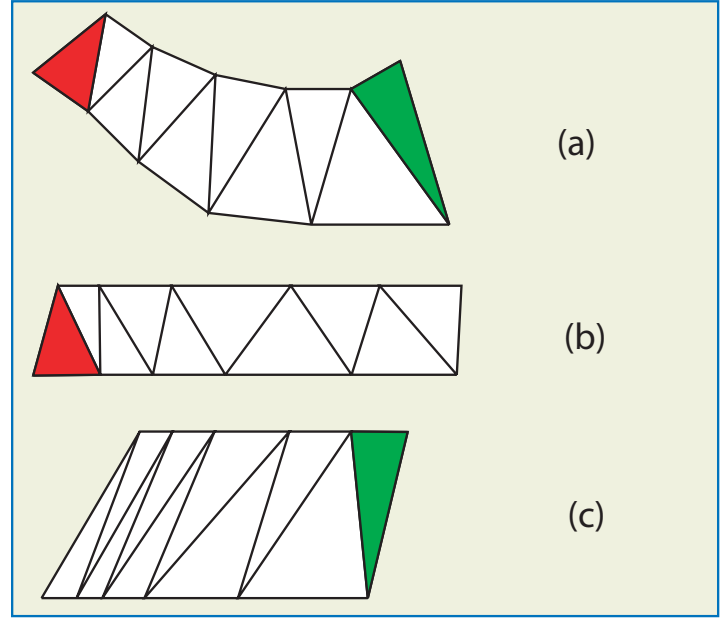

Figure 6: Surface flattening: curved mesh in 3D (a). Depending on the choice of the focal triangle, i.e., red, or green, the patch is growing, by preserving the alignment of the points in contours and minimizing the area distortion (b),(c).

to the neighboring contour. The distance between these two slices is defined by the height of the focal triangle (height $=\frac{2 \cdot \text { area }}{|p 2-p 1|}$ ) (see figure 4). Moreover, we define as an active set those points which have not been added to the patch yet but are forming a triangle with two points on the boundary of the patch. In the next step the patch is iteratively flattened by adding active points $a_{i}$ to the patch. Positions of $a_{i}$ are selected on the line corresponding to a contour so that, for example, the area of the currently flattened triangle is preserved. In this way, in each step a triangle nearest to the focal point is newly added to the patch. Note that arrangement of the points in the contours prevents local as well as global intersections. Any other surface parameterization method, based on different constrains, can be performed in a similar way. The selection of the focal triangle is performed during the inspection by a mouse click on the surface of the height field. Notice, that, due to the above mentioned alignment of the processed points, the distortion minimization for a new focal triangle yields an interactive feedback. Thus, the user is able to investigate all suspicious areas of the cartilage within several seconds.

For the sake of clarity, we illustrate the method on a simple example. Assume, we want to minimize the area distortion. Fig-

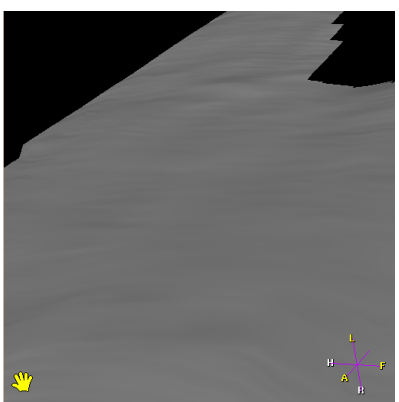

$(a)$

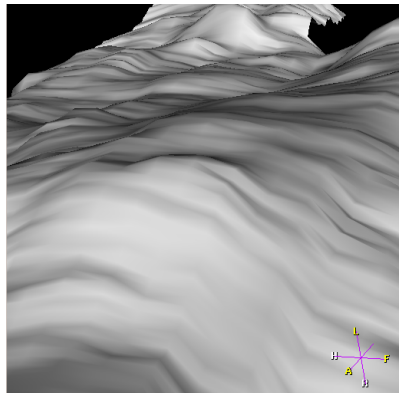

$(b)$
Figure 7: A detailed cartilage surface without (a) and with (b) nonlinear scaling. The roughness of the surface is hardly noticeable without scaling.

ure 6 demonstrates the difference between selecting two different focal triangles (red or green, see figure 6 (a)). The selected triangle is rigidly transformed to the patch and determines the distance between the transformed contours (see figure 6 (b),(c)). Further added triangles preserve their area by changing the distance inside the contour.

\section{Operations on the Height Field}

The planar representation of the curved cartilage surface enables effective visualization of its thickness. Slight changes in the thickness on the reconstructed surface may, however, not be noticeable (see figure 7 (a)). The intuitive approach - uniform scaling will not bring any improvement. Since we would like to enhance the thickness information, we propose a non-uniform scaling by applying scaling only in the height direction (see figure 7 (b)). This has already been done for earth visualizations to emphasize topographic variations like mountains and valleys. Note, that the below described operations, i.e., non-uniform scaling, may lead to intersections for non-convex surfaces. The height field representation of the thickness information does not have to deal with this problem. The triangulated surface is flattened into the 2D plane and the thickness is mapped to the third coordinate. Thus, the height field can be scaled in the thickness direction without distorting the thickness values (see figure 5).

Similarly, any two-dimensional technique can be applied in order to visualize scalar or vector values on the curved surface. To illustrate the wide application area we discuss several visualization 


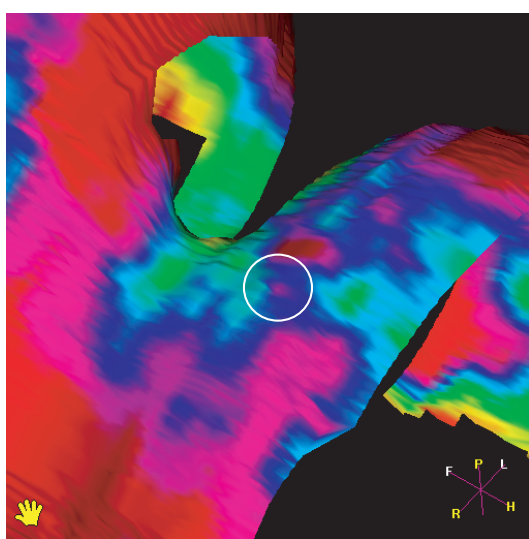

(a)

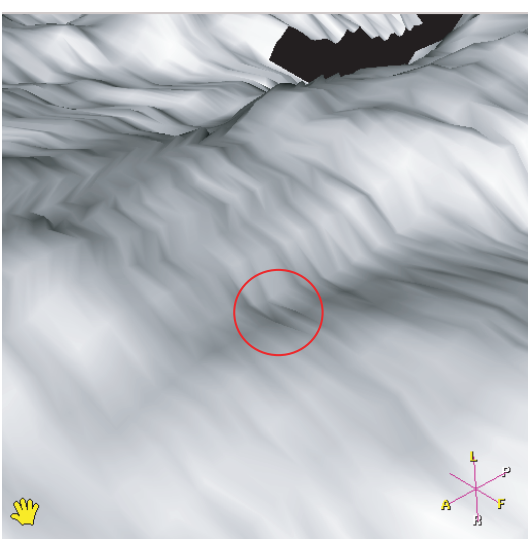

(b)

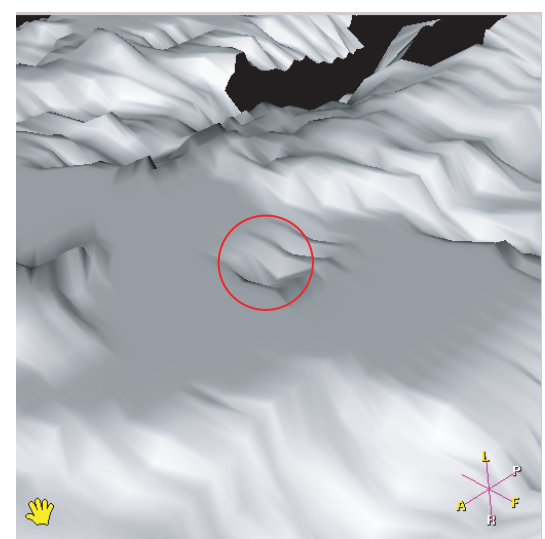

(c)

Figure 8: Thresholded scaling: The scaling factor can be set independently for each thickness interval. This allows to flatten the values which have no importance for the inspection, while scaling only the values below and above the value range, respectively: reconstructed surface (a), non-uniformly scaled surface (b), thresholded scaling with three intervals (low, middle, high) (c). Flattening of the middle values, allows the user to concentrate on the areas with the suspicious values (circle).

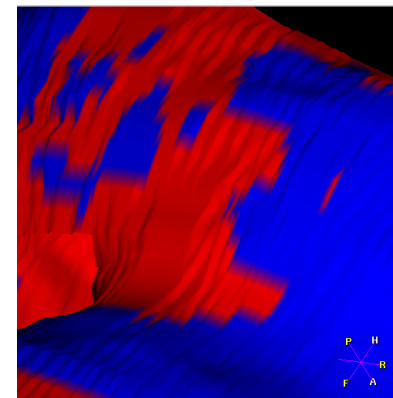

(a)

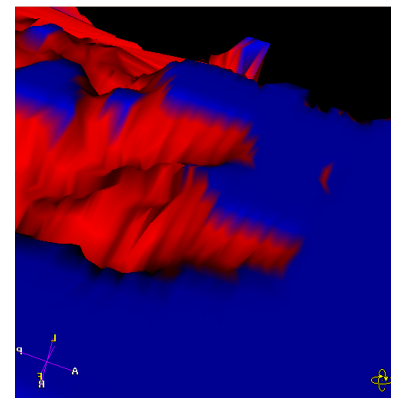

(b)

Figure 9: Thresholded non-linear scaling: the color map on the reconstructed surface depicts areas on the cartilage surface with thickness below a certain threshold (red), while the remaining part is mapped to blue (a). The non-linear scaling enables a more detailed view of the thickness changes in the thin area (b).

techniques for height fields, which exploit the flattening of a curved surface.

\subsection{Thresholded Non-linear Scaling}

In the case of cartilage visualization, we are interested in areas where the cartilage is thinning. Therefore, we want to inspect those areas, where the thickness is below a certain threshold. When scaling the entire height field, the enhancement of areas which are of no interest may disturb the inspection, or hide the parts of the height field where the thickness is rather low. This is especially true if the variation of the thickness, which is of interest, is relatively small as compared to the overall thickness range. Therefore, in addition to the non-uniform scaling, we propose a thresholded non-linear scaling (see figure 9). The thresholding of the values above the predefined threshold will flatten the high values, thus, performing the scaling only on the values below the threshold. Figure 10 (a) illustrates this concept.

\subsection{Non-linear Scaling on Interval}

A natural extension to the thresholded non-linear scaling is the scaling on a certain range of thickness values. An arbitrary number of

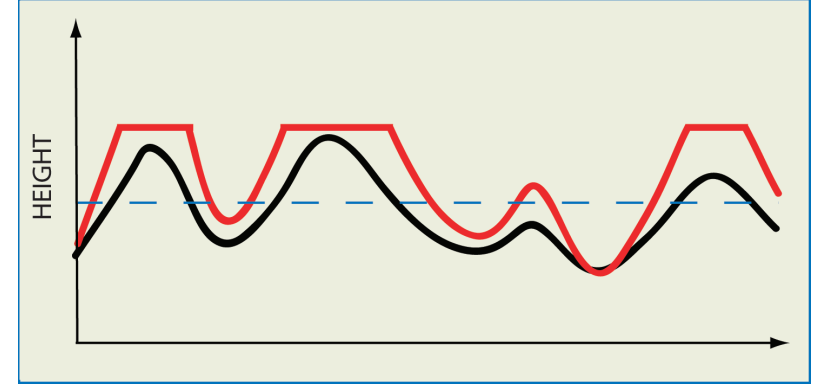

(a)

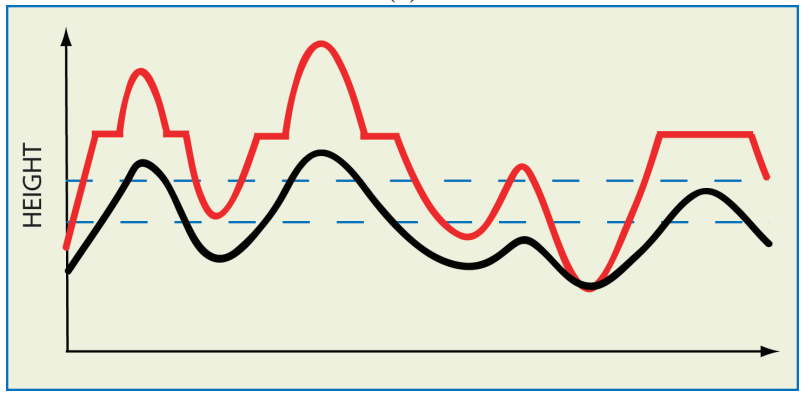

(b)

Figure 10: Sketch of the thresholded non-linear scaling (a), and the non-linear scaling on an interval (b). The original function is depicted in black, while the scaled function is depicted in red. The blue dotted lines represent the thresholds.

value ranges can be defined in order to perform custom scaling for each interval. Figure 8 illustrates a case with three height intervals. Assume, we are interested only in the pathologic cases, i.e., where the thickness is below one threshold and above another threshold. Three intervals are defined for low, middle and high thickness values. A linear scaling can be defined for each interval, respectively. Setting the scaling factor to zero for the values in the range between the two thresholds, allows the user to concentrate on the areas with the suspicious/specific values (circle) (see figure 8 (c)). The idea is sketched in figure 10 (b).

The following examples show the extraction of the thickness information enhanced by color coding (see figure 11), by iso-lines 


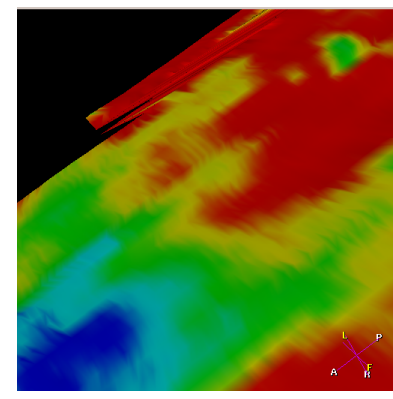

(a)

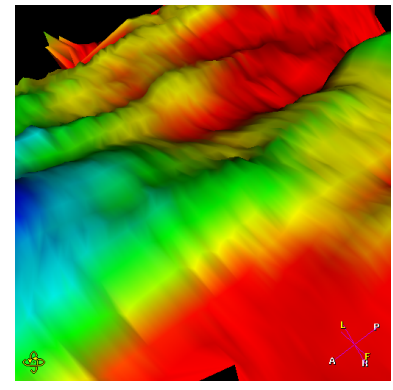

(b)
Figure 11: Surface of the cartilage without (a) and with the nonlinear scaling (b). By increasing the scale factor it is possible to inspect also tiny changes in the thickness of the tissue.

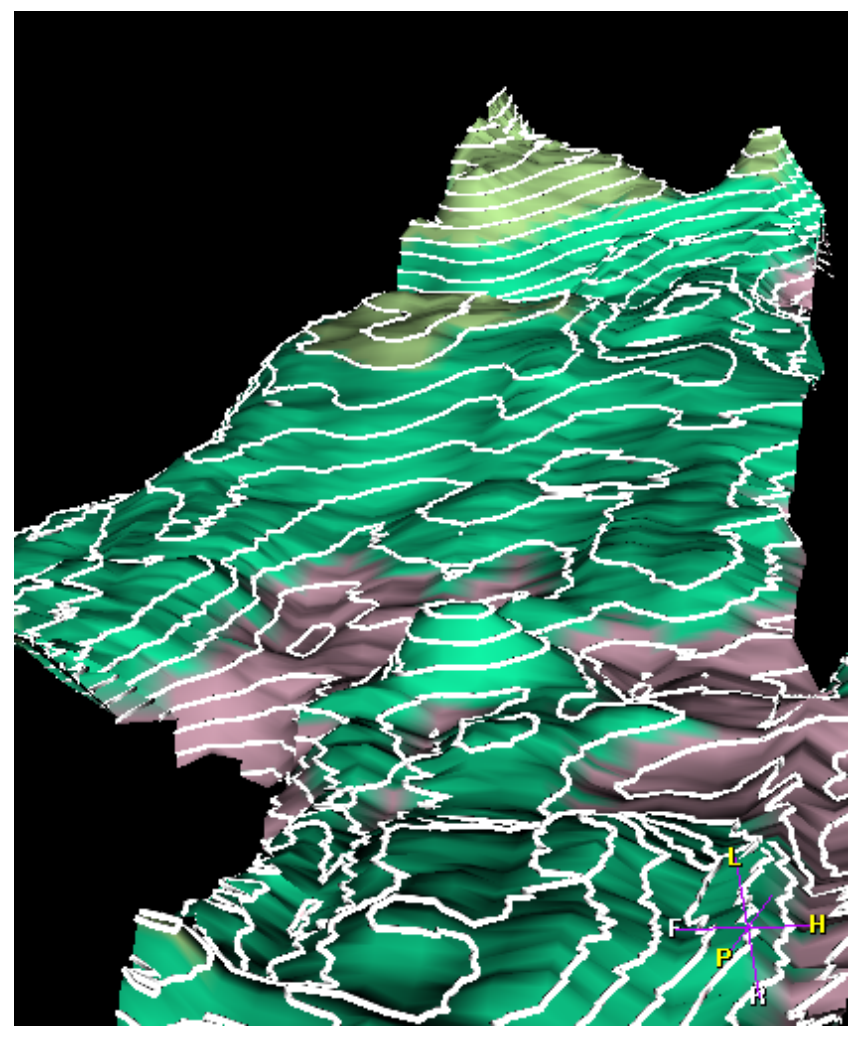

Figure 12: Height field representation of the cartilage thickness enhanced by iso-lines.

(see figure 12), and by glyphs (see figure 13). These representations of the unfolded cartilage yield additional information to the visualization, e.g., absolute thickness, or thickness gradient magnitude.

\subsection{Scale Transfer Function}

As mentioned above the overall thickness of the cartilage varies from patient to patient. Thus, we need a tool which enables detection of subtle thickness changes on each range of the thickness values. Using the basic non-linear scaling approach, interesting features may be occluded by other scaled areas, which are not of interest. This drawback can be overcome by generalizing the thresholded non-linear scaling. We define a continuous piecewise linear scaling transfer function [4], which maps the original thickness val-

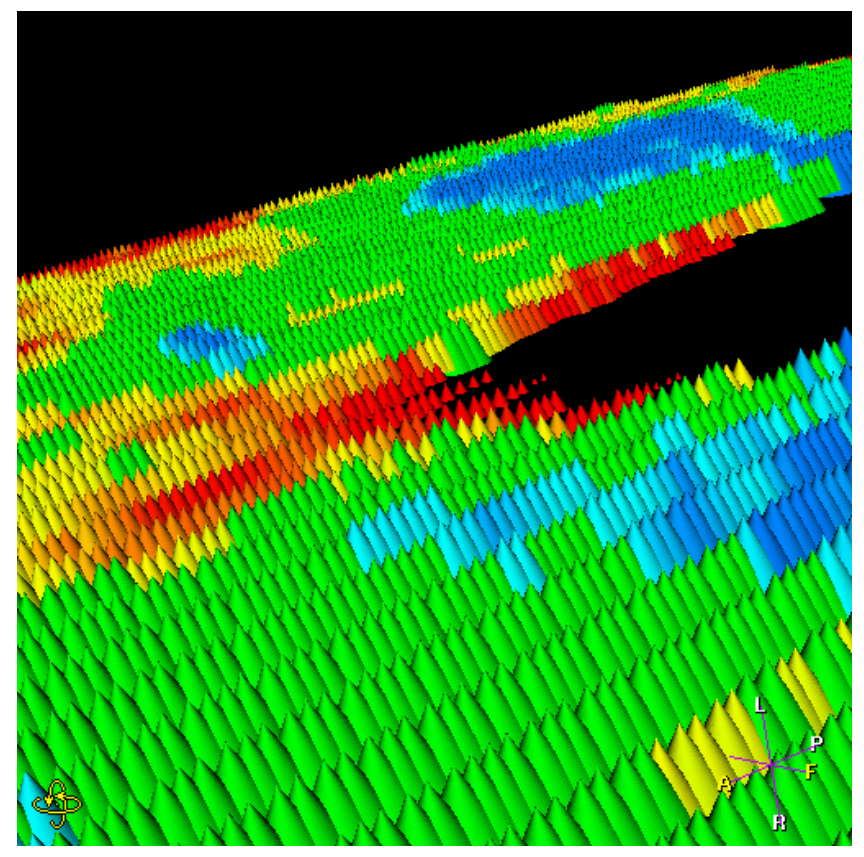

Figure 13: Glyph representation of the surface thickness: size of the glyph increases with the thickness value.

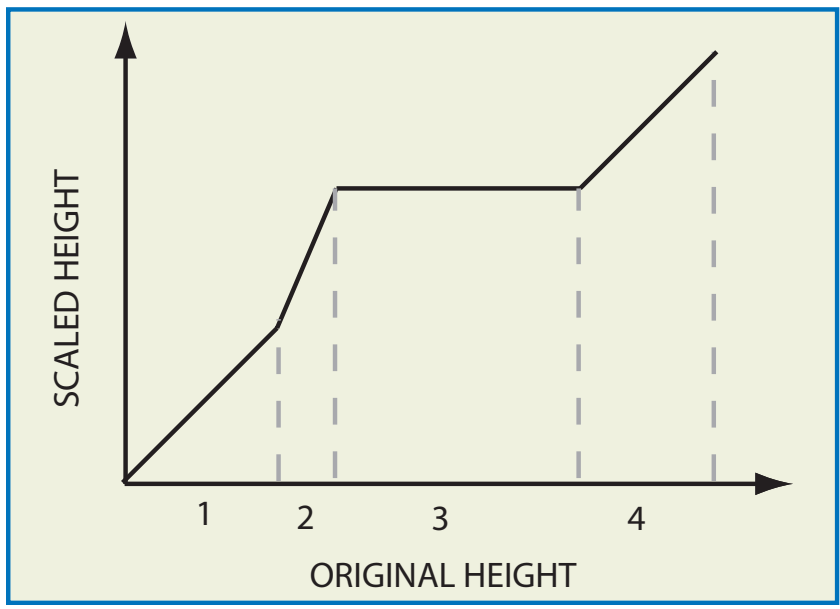

Figure 14: An example of a simple scale transfer function. The scaling is performed on interval 2 , while the values within the intervals 1 and 4 preserve the original value. The mapping of the values belonging to interval 3 flattens the surface in this area.

ues, assigned to each vertex, to the scaled values (see figure 14). Note, that the intervals parallel to the $x$-axis correspond to flattening of the field (see figure 14, interval 3). Thickness preservation is performed on intervals, where $\triangle x=\triangle y$ (see figure 14, interval 1 and 4).

\section{Summary and Conclusions}

We have presented a method to visualize the thickness of curved thin objects. The approach has been illustrated on the visualization of articular cartilage. This is a structure where the detection of slight thickness changes is vital for diagnosis. It has been shown that unfolding of anatomic organs is promising since it enables 
the application of 2D visualization methods. Application of these methods is not possible on the curved reconstructed surfaces.

The above described work has been implemented as a part of a framework for cartilage visualization. It includes several linked views, which allow inspection of the articular cartilage with backcoupling to the reconstructed surface as well as to the original slices.

The future work on articular cartilage visualization will continue with a broader clinical study on a variety of datasets. This will include experimental validation and proof of the usefulness of the applied methods on a couple of scenarios from different modalities. Furthermore, in the scope of the project we aim for faster and more robust segmentation methods.

\section{ACKNOWLEDGEMENTS}

This work has been funded by Philips Medical Systems in the scope of the COMRADE project (MRI based Visualization and Analysis for Virtual Colonoscopy and Orthopaedics). We thank Frans Gerritsen and Pierre Ermes of Philips Medical Systems and Rob van der Rijt and Harrie van den Bosch of Catharina Hospital in Eindhoven for their collaboration.

\section{REFERENCES}

[1] S. Angenent, S. Haker, A. Tannenbaum, and R. Kikinis. On the Laplace-Beltrami operator and brain surface flattening. IEEE Transactions on Medical Imaging, 18:700-711, 1999.

[2] A. Vilanova Bartrolí, R. Wegenkittl, A. König, and E. Gröller. Nonlinear virtual colon unfolding. In IEEE Visualization 2001, pages 411 418, 2002.

[3] Ch. Bennis, J.-M. Vézien, and G. Iglésias. Piecewise surface flattening for non-distorted texture mapping. In SIGGRAPH 1991, pages 237246, 1991.

[4] S. K. Card, J. D. Mackinlay, and B. Shneiderman. Readings in Information Visualization (Using Vision to Think). Morgan Kaufman Publishers, Inc., 1999.

[5] P. Danielsson. Euclidean distance mapping. Computer Graphics and Image Processing, 14:227-248, 1980.

[6] D. G. Disler, M. P. Recht, and T. R. McCauley. MR imaging of articular cartilage. Skeletal Radiology, 29:367-377, 2000.

[7] A. B. Ekoule, F. C. Peyrin, and C. L. Odet. A triangulation algorithm from arbitrary shaped multiple planar contours. ACM Transactions on Graphics, 10(2):182-199, 1991.

[8] B. Fischl, M. I. Sereno, and A. M. Dale. Cortical surface-based analysis II: Inflation, flattening, and a surface-based coordinate system. NeuroImage, 9:195-207, 1999.

[9] M. S. Floater and K. Hormann. Surface parameterization: a tutorial and survey. In Advances on Multiresolution in Geometric Modelling, to appear. Springer, 2004.

[10] A. Guermazi, S. Zaim, B. Taouli, Y. Miaux, Ch. G. Peterfy, and H. K. Genant. MR findings in knee osteoarthritis. European Radiology, 13:1370-1386, 2003.

[11] S. Haker, S. Angenent, A. Tannenbaum, and R. Kikinis. Nondistorting flattening maps and the $3 \mathrm{D}$ visualization of colon $\mathrm{CT}$ images. IEEE Transactions on Biomedical Engineering, 19(7):665-671, 2000.

[12] F. Heuer, M. Sommers, J. B. Reid III, and M. Bottlang. Estimation of cartilage thickness from joint surface scans: Comparative analysis of computational methods. In ASME, pages 569-570, 2001.

[13] H. Imhof, I.-M. Nöbauer-Huhmann, C. Krestan, A. Gahleitner, I. Sulzbacher, S. Marlovits, and S. Trattnig. MRI of the cartilage. European Radiology, 12:2781-2793, 2002.

[14] A. Kanitsar, D. Fleischmann, R. Wegenkittl, P. Felkel, and M. E. Gröller. CPR - Curved Planar Reformation. In IEEE Visualization 2002, pages 37-44, 2002.
[15] M. Kass, A. Witkin, and D. Terzopoulos. Snakes: Active contour models. International Journal of Computer Vision, 1(4):321-331, 1987.

[16] B. Lévy and J.-L. Mallet. Non-distorted texture mapping for sheared triangulated meshes. In SIGGRAPH 1998, pages 343-352, 1998.

[17] S. Lobregt and M.A. Viergever. A discrete dynamic contour model. IEEE Transactions on Medical Imaging, 14(1):12-24, March 1995.

[18] J. C. Mullikin. The vector distance transform in two and three dimensions. GMIP, 54(6):526-535, November 1992.

[19] A. W. Paeth. Digital cartography for computer graphics. Graphics Gems, pages 307-320, 1990.

[20] P. V. Sander, J. Snyder, S. Gortler, and H. Hoppe. Texture mapping progressive meshes. In SIGGRAPH 2001, pages 409-416, 2001.

[21] R. Satherley and M. W. Jones. Vector-city vector distance transform. Computer Vision and Image Understanding, 82(3):238254(17), 2001.

[22] O. Sorkine, D. Cohen-Or, R. Goldenthal, and D. Lischinski. Boundeddistortion piecewise mesh parameterization. In IEEE Visualization 2002, pages 355-362, 2002.

[23] T. G. Williams, Ch. J. Taylor, Z. Gao, and J. C. Waterton. Corresponding articular cartilage thickness measurements in the knee joint by modelling the underlying bone. In IPMI, volume 2732 , pages 126 $135,2003$. 